\title{
Incidência de doença diarreica aguda em Caxias do Sul, Rio Grande do Sul, Brasil, em uma série histórica de 10 anos
}

\author{
Incidence of acute diarrheal disease in Caxias do Sul, Rio Grande do Sul, Brazil, \\ in a 10-year historical series
}

\author{
Suelen Paesi ${ }^{1} \bowtie$, Flaviane Eva Magrini² $^{2}$ \\ ${ }^{1}$ Bióloga. Doutora em Ciências Biológicas pela Universidade Federal do Rio Grande do Sul (UFRGS). Coordenadora do Laboratório de Diagnóstico Molecular, \\ Instituto de Biotecnologia, Universidade de Caxias do Sul (UCS), Caxias do Sul, RS. \\ 2 Bióloga. Mestre e doutoranda em Biotecnologia pela UCS. Atua no Laboratório de Diagnóstico Molecular, Instituto de Biotecnologia, UCS, Caxias do Sul, RS.
}

\section{RESUMO}

Objetivos: Avaliar a incidência de doença diarreica aguda registrada em Caxias do Sul, em um período de 10 anos, investigando suas correlações com a vacinação contra rotavírus, com a precipitação pluviométrica e com o número de internações hospitalares.

Métodos: Foi realizado um estudo descritivo dos registros de doença diarreica aguda, disponibilizados pelo setor de Vigilância Epidemiológica da Secretaria Municipal de Saúde de Caxias do Sul.

Resultados: Entre 2004 e 2013 foram registrados 61.246 casos de doença diarreica aguda, sendo que os maiores números ocorreram em bairros de baixa renda. Durante o período de avaliação foram identificados cinco surtos epidêmicos de doença diarreica aguda, em meses de verão e inverno. Não foram encontradas relações entre doença diarreica aguda e precipitação pluviométrica. O número de internações hospitalares por doença diarreica aguda em crianças de zero a quatro anos decresceu a partir de 2006, coincidindo com o início do programa de vacinação contra rotavírus na rede pública, enquanto que os óbitos de todas as idades sofreram flutuações durante o período estudado.

Conclusões: A avaliação da incidência de doença diarreica aguda, por longo período, mostra que houve flutuações ao longo do tempo no número de registros e de óbitos em Caxias do Sul. Este estudo revelou também uma redução no número de internações devidas a doença diarreica aguda no período que sucedeu à introdução do programa de vacinação contra rotavírus na rede pública de saúde.

DESCRITORES: diarreia; rotavírus; vacinas; incidência; hospitalização; pluviometria; pluviosidade.

\section{ABSTRACT}

Aims: To evaluate the incidence of acute diarrheal disease (ADD) recorded over a 10-year period in Caxias do Sul, southern Brazil, and its correlations with the vaccination against rotavirus, with rainfall, and with the number of hospitalizations.

Methods: A descriptive study was conducted, analyzing the ADD records obtained from the Epidemiological Surveillance sector of the Local Health Department of Caxias do Sul.

Results: A total of 61,246 cases of ADD were recorded between 2004 and 2013, most of which occurred in low-income neighborhoods. There were five outbreaks of ADD in the summer and winter months during the study period. No correlations were found between ADD and rainfall. The number of children aged between zero and four years hospitalized for ADD decreased from 2006 onwards, when vaccination against rotavirus was implemented in the public health network whereas the number of deaths across all age groups fluctuated during the study period Conclusions: The assessment of the long-term incidence of ADD showed a fluctuation in the number of cases and deaths in Caxias do Sul. In addition, hospitalizations for ADD decreased after the implementation of rotavirus vaccination in the public health network.

KEY WORDS: diarrhea; rotavirus; vaccines; incidence; hospitalization; pluviometry; rainfall amount. 
Abreviatura: DDA, doença diarreica aguda.

\section{INTRODUÇÃO}

A doença diarreica aguda (DDA) é um agravo dos mais comuns no mundo inteiro, sendo a segunda causa de morbimortalidade infantil [1]. Todos os anos, aproximadamente dois milhões de crianças morrem por causa das complicações ocasionadas pelas doenças diarreicas em países subdesenvolvidos [2,3,4].

As complicações mais frequentes da DDA são a desidratação e a desnutrição, podendo levar ao óbito. Os episódios diarreicos repetidos podem ocasionar desnutrição crônica, com consequências no desenvolvimento físico e mental $[5,6]$. Em adultos, apesar da baixa mortalidade, a gastroenterite leva a sintomas que afastam os pacientes dos seus postos de trabalho, gerando elevadas perdas aos setores privados e altos custos aos órgãos públicos de saúde [7].

A DDA envolve uma diversificada gama de agentes etiológicos, como vírus, bactérias e parasitas [8]. A prevalência dos patógenos varia de acordo com as condições de vida da população, recursos econômicos, nutricionais, localização geográfica, faixa etária e sazonalidade $[9,10]$. Os agentes virais são mais frequentes no mundo todo, enquanto que as infecções bacterianas são relevantes em países com limitado saneamento básico [11] e população com nutrição precária. Entre as bactérias causadoras de diarreia podem ser citadas Escherichia coli, Campylobacter sp, Shigella sp e Salmonella sp [12]. Os principais agentes virais são rotavírus, norovírus, astrovírus e adenovírus [13], e os agentes parasitários mais frequentes são Cryptosporidium spp, Giardia lamblia e Ascaris sp [14]. Em 2011, os indicadores de mortalidade mostraram 756 óbitos por DDA, no Brasil, sendo 10 casos no Rio Grande do Sul [15]. Embora não exista um recurso preventivo eficaz para todos os agentes gastroentéricos vinculados à diarreia, em 2006 foi introduzida a vacina contra rotavírus no calendário infantil. Estudos mostram que o programa associouse a uma redução nas taxas de infecção (7,4 a 77\%), de hospitalização (12,3 a 55,4\%) e de mortalidade (22 a 50\%) infantil em todo o território brasileiro [16].

A Cidade de Caxias do Sul está localizada ao sul do Brasil, na região da Serra Gaúcha, possuindo uma população estimada em 470 mil habitantes segundo o Instituto Brasileiro de Geografia e Estatística @2014 [17]. O clima da cidade é temperado, com verões amenos, invernos com aproximadamente 463 horas de frio (maio-setembro) e precipitações pluviométricas regularmente distribuídas durante o ano [18]. A cidade é conhecida por ser um dos parques da indústria metalmecânica mais importantes do País, tendo um dos maiores índices de desenvolvimento socioeconômico do Estado do Rio Grande do Sul. O município está dividido oficialmente em 65 bairros e conta com 47 unidades de saúde de pronto atendimento e sete hospitais. Em Caxias do Sul, não são conhecidos números de DDA registrados por longos períodos. Essa informação poderia mostrar o impacto da vacinação contra rotavírus sobre o agravo, e também serviria para nortear políticas públicas e privadas de saúde coletiva.

Diante disso, este trabalho teve por objetivo avaliar a incidência de DDA registrada no Município de Caxias do Sul, por um período de 10 anos (2004-2013), a fim de correlacionar este agravo com a precipitação pluviométrica, com o número de internações hospitalares e com a cobertura vacinal contra rotavírus no município.

\section{MÉTODOS}

Este trabalho consiste em um levantamento do número de registros de DDA total e por bairro (conforme as Unidades de Pronto Atendimento); do número de internações hospitalares (conforme o Sistema de Internação Hospitalar) de crianças de zero a quatro anos, no período pré e pós-vacinal (ou seja, antes e depois de ser disponibilizada a vacina contra rotavírus na rede pública de saúde); do percentual da cobertura vacinal contra rotavírus entre 2006 e 2013 (conforme o Programa Nacional de Imunizações); e do número de óbitos por DDA (conforme o Sistema de Informação sobre Mortalidade) em todas as faixas etárias, disponibilizados pelo setor de Vigilância Epidemiológica da Secretaria Municipal de Saúde de Caxias do Sul, Rio Grande do Sul, relativos ao período de 2004 a 2013.

Foi aplicado o teste de Pearson para avaliar as correlações entre surtos epidêmicos de DDA e pluviosidade, e entre número de internações hospitalares e cobertura vacinal para rotavírus. Para o cálculo de incidência foi utilizado o número de registro de DDA em cada mês/ano, de acordo com o número de habitantes no período, dividido por 100.000 habitantes. O coeficiente de mortalidade foi calculado a partir do número de óbitos anuais por DDA/número de habitantes no período, multiplicando-se por 1.000 . $\mathrm{O}$ número de habitantes utilizado para o cálculo da incidência e do coeficiente de mortalidade foi estimado pelo IBGE, baseado no censo e estimativas de 2000, 2010 e 2014 [17]. Os índices pluviométricos foram 
informados pelo Centro Estadual de Meteorologia do Rio Grande do Sul. Para a identificação dos surtos de DDA foram usados os limites inferiores e superiores calculados a partir da média de cada mês ao longo de 10 anos (média $\pm 1,96$ desvios padrão). A tabulação e análise dos dados foi realizada por meio do programa Excel 2010 e SPSS 21.

\section{RESULTADOS}

No período de 2004 a 2013 foram registrados 61.246 casos de DDA no Município de Caxias do Sul, com uma média anual de 6.124 notificações. A partir do ano de 2006, ocorreu um aumento das notificações, sendo 2010 o ano com a maior incidência cumulativa de DDA (1.810 para cada 100.000 habitantes), com 7.877 casos registrados, seguido pelo ano de 2008 , com incidência cumulativa anual de 1.630 , e 2012 e 2013 , com 1.551 e 1.533 , respectivamente. O menor número de registros e de incidência anual de DDA foi verificado no ano de 2005. A incidência cumulativa no período pré-vacinal (anterior a 2006) pouco difere da registrada no período pós-vacinal (após 2006) (Tabela 1).

Verificou-se que as gastroenterites ocorreram durante todos os meses de todos os anos avaliados (Tabela 1). Contudo, foram registrados cinco picos epidêmicos de DDA durante o período analisado. Os surtos ocorreram nos meses de setembro de 2004, com uma incidência de 225,3 para cada 100.000 habitantes; março e dezembro de 2010 (respectivamente 230/100.000 e 280/100.000); janeiro de 2011 (211,9/100.000); e um surto com duração de dois meses entre maio $(230 / 100.000)$ e junho $(186,7 / 100.000)$ de 2012 (Figura 1).

Tabela 1. Incidência de doença diarreica aguda por mês e ano (2004 a 2013) no Município de Caxias do Sul, Rio Grande do Sul, Brasil.

\begin{tabular}{|c|c|c|c|c|c|c|c|c|c|c|c|c|}
\hline Mês & 2004 & 2005 & 2006 & 2007 & 2008 & 2009 & 2010 & 2011 & 2012 & 2013 & Média & DP \\
\hline Jan & 123,0 & 99,74 & 93,06 & 145,11 & 174,26 & 129,98 & 136,32 & 211,99 & 97,33 & 81,29 & 129,2 & 40,43 \\
\hline Fev & 104,0 & 75,52 & 111,57 & 137,59 & 122,79 & 121,10 & 193,33 & 178,51 & 120,67 & 74,62 & 124,0 & 38,48 \\
\hline Mar & 121,4 & 116,15 & 120,57 & 154,14 & 110,05 & 113,67 & 230,57 & 192,31 & 100,00 & 101,72 & 136,1 & 43,38 \\
\hline $\mathrm{Abr}$ & 67,8 & 72,40 & 89,20 & 144,61 & 143,14 & 142,21 & 101,38 & 101,58 & 146,89 & 148,39 & 115,8 & 32,67 \\
\hline Maio & 59,6 & 77,34 & 89,72 & 110,53 & 101,72 & 107,19 & 82,76 & 80,09 & 230,00 & 193,12 & 113,2 & 54,71 \\
\hline Jun & 74,9 & 116,15 & 66,84 & 60,65 & 107,35 & 87,77 & 128,97 & 74,89 & 186,67 & 115,48 & 102,0 & 37,89 \\
\hline Jul & 72,3 & 96,61 & 86,89 & 61,15 & 145,59 & 96,64 & 124,37 & 63,35 & 164,89 & 152,04 & 106,4 & 38,00 \\
\hline Ago & 215,3 & 117,45 & 183,55 & 86,47 & 165,20 & 97,36 & 88,05 & 75,79 & 172,67 & 109,46 & 131,1 & 48,80 \\
\hline Set & 225,3 & 48,70 & 173,01 & 90,73 & 139,46 & 133,09 & 118,85 & 67,87 & 97,33 & 106,02 & 120,0 & 51,45 \\
\hline Out & 148,5 & 60,68 & 132,13 & 146,62 & 174,02 & 139,57 & 125,29 & 90,72 & 116,89 & 138,92 & 127,3 & 31,98 \\
\hline Nov & 111,1 & 92,45 & 136,76 & 138,60 & 102,21 & 173,38 & 200,00 & 97,74 & 54,89 & 155,27 & 126,2 & 42,97 \\
\hline Dez & 137,5 & 84,90 & 111,83 & 140,35 & 144,85 & 193,05 & 280,92 & 147,06 & 63,56 & 157,20 & 146,1 & 59,95 \\
\hline $\begin{array}{l}\text { Incidência } \\
\text { cumulativa }\end{array}$ & 1.460 & 1.058 & 1.395 & 1.416 & 1.630 & 1.535 & 1.810 & 1.381 & 1.551 & 1.533 & - & - \\
\hline
\end{tabular}

Os números representam a incidência por 100.000 habitantes. DP: desvio padrão.

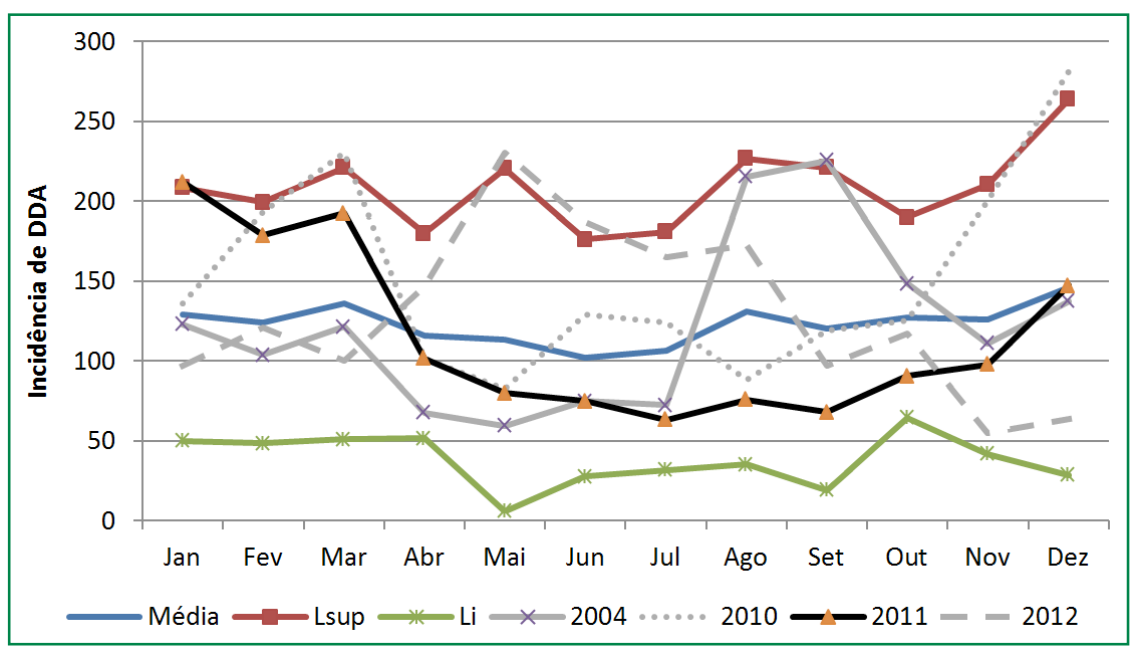

Figura 1. Incidência de surtos epidêmicos de doença diarreica aguda no Município de Caxias do Sul, Rio Grande do Sul, Brasil, 2004-2013. A incidência é representada em números por cada 100.000 habitantes.

DDA: doença diarreica aguda; Lsup: limite superior; Li: limite inferior. 
A análise da correlação entre incidência de surtos epidemiológicos de DDA e pluviosidade do município mostrou uma correlação inversa moderada $(r=-0,43)$, porém não significativa $(\mathrm{p}=0,64)$, em todo o período de 10 anos de avaliação (Figura 2).

As maiores ocorrências de DDA foram registradas na região oeste da cidade, no bairro Reolon, por quatro anos consecutivos, de 2007 a 2010, sendo que em 2008 registraram-se 397 casos, representando 5,9\% do número total de registros no ano (6.653). Em seguida, aparece o bairro Desvio Rizzo, na região sudoeste do município, em 2005, 2012 e 2013, com 188, 331 e 307 casos, respectivamente e, o bairro Santa Fé, na área norte, em 2006 e 2011 (Figura 3).
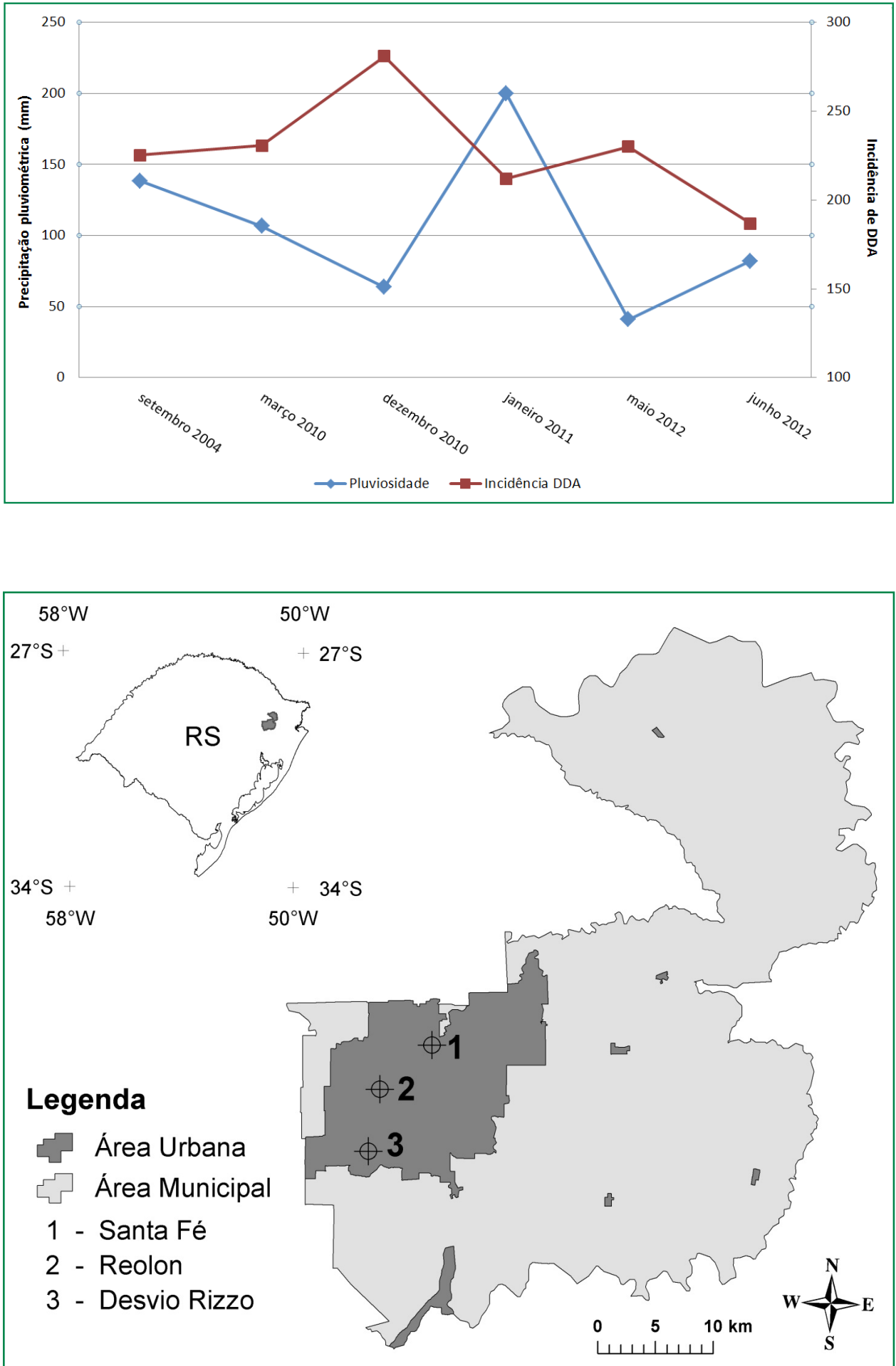

Figura 2. Incidência de surtos epidêmicos de doença diarreica aguda e pluviosidade (mm) no Município de Caxias do Sul, Rio Grande do Sul, Brasil, 2004-2013. O teste de Pearson mostra uma correlação inversa moderada $(r=-0,43)$ entre as duas variáveis, porém não significativa $(p=0,64)$. $A$ incidência é representada em números por cada 100.000 habitantes.

DDA: doença diarreica aguda.
Figura 3. Mapa da localização dos bairros com mais registros de casos de doença diarreica aguda no período de 2004 a 2013 no Município de Caxias do Sul, Rio Grande do Sul, Brasil. 
O número de internações hospitalares de crianças de um a quatro anos foi $110 \%$ maior que o da faixa etária abaixo de um ano de idade em um período de dez anos. O ano de 2005 teve o maior número

Tabela 2. Internações hospitalares de crianças de menos de cinco anos com doença diarreica aguda no Município de Caxias do Sul, Rio Grande do Sul, Brasil, 2004-2013.

\begin{tabular}{|c|r|r|r|r|r|r|}
\hline \multirow{2}{*}{ Ano } & \multicolumn{2}{|c|}{$<\mathbf{1}$ ano } & \multicolumn{2}{c|}{1 a 4 anos } & \multicolumn{2}{c|}{$\begin{array}{c}\text { Total de } \\
\text { internações }\end{array}$} \\
\cline { 2 - 7 } & $\mathbf{N}$ & $(\%)$ & \multicolumn{1}{c|}{$\mathbf{N}$} & \multicolumn{1}{c|}{$(\%)$} & \multicolumn{1}{c|}{$\mathbf{N}$} & $(\%)$ \\
\hline 2004 & 80 & $(27,2)$ & 214 & $(72,8)$ & 294 & $(100)$ \\
\hline 2005 & 90 & $(26,4)$ & 251 & $(73,6)$ & 341 & $(100)$ \\
\hline 2006 & 74 & $(23,1)$ & 246 & $(76,8)$ & 320 & $(100)$ \\
\hline 2007 & 30 & $(18,3)$ & 134 & $(81,7)$ & 164 & $(100)$ \\
\hline 2008 & 63 & $(26,8)$ & 172 & $(73,2)$ & 235 & $(100)$ \\
\hline 2009 & 58 & $(36,9)$ & 99 & $(63,1)$ & 157 & $(100)$ \\
\hline 2010 & 84 & $(34,7)$ & 158 & $(65,3)$ & 242 & $(100)$ \\
\hline 2011 & 46 & $(68,6)$ & 21 & $(31,4)$ & 67 & $(100)$ \\
\hline 2012 & 116 & $(69,04)$ & 52 & $(30,96)$ & 168 & $(100)$ \\
\hline 2013 & 24 & $(31,57)$ & 52 & $(68,42)$ & 76 & $(100)$ \\
\hline Total & 665 & $(32,2)$ & 1.399 & $(67,8)$ & 2.064 & $(100)$ \\
\hline
\end{tabular}

de internações, com 251 registros para a faixa de um a quatro anos, representando $73,6 \%$ do total de internações dessas crianças (Tabela 2). Também se verificou que o percentual de internações de crianças de um a quatro anos foi diminuindo a partir de 2006, sendo que os menores números de internações por causa de diarreia foram registrados nos anos de 2011 (21 casos), 2012 e 2013 (52 casos cada).

Em geral, observa-se um decréscimo do número de internações hospitalares por DDA de crianças de zero a quatro anos no período pós-vacinal, verificando-se uma correlação inversa $(r=-0,67)$ entre o número de internações e a cobertura vacinal contra rotavírus no Município de Caxias do Sul (Figura 4). Após o ano de 2007, ocorreu um aumento da cobertura vacinal de $61,5 \%$ para $90,3 \%$, que se manteve crescente até 2013 .

No monitoramento de 10 anos de estudo, registraram-se 55 óbitos por DDA, em todas as idades, demonstrando uma flutuação nos coeficientes de mortalidade por este agravo (Figura 5). Os maiores coeficientes de mortalidade foram registrados em 2005 $(0,0208), 2011(0,0180)$ e $2013(0,0171)$, enquanto que no ano de 2007 registrou-se o menor coeficiente $(0,0050)$.

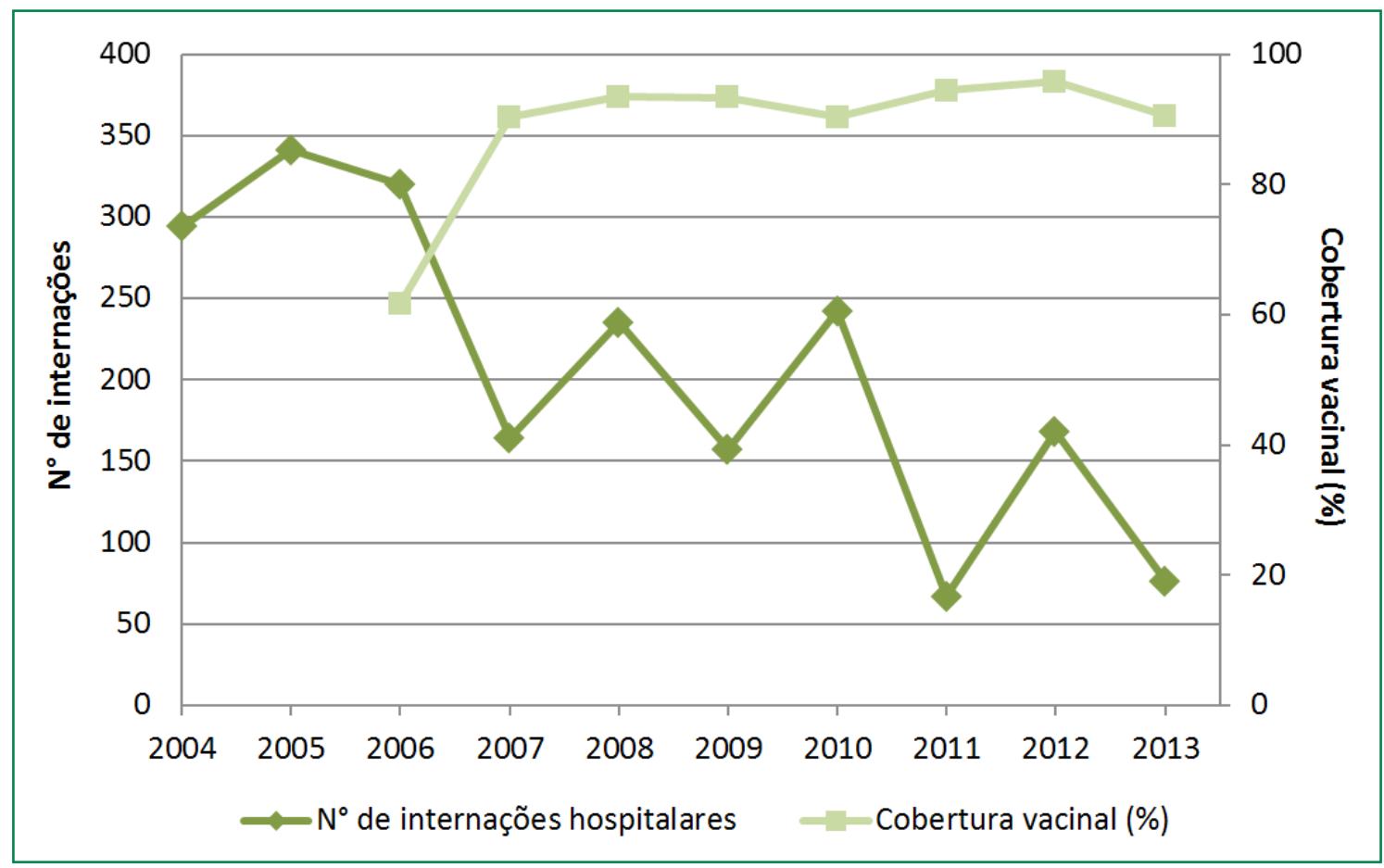

Figura 4. Número de internações hospitalares de crianças de zero a quatro anos por doença diarreica aguda e cobertura vacinal contra rotavírus no Município de Caxias do Sul, Rio Grande do Sul, Brasil, 2004-2013. 


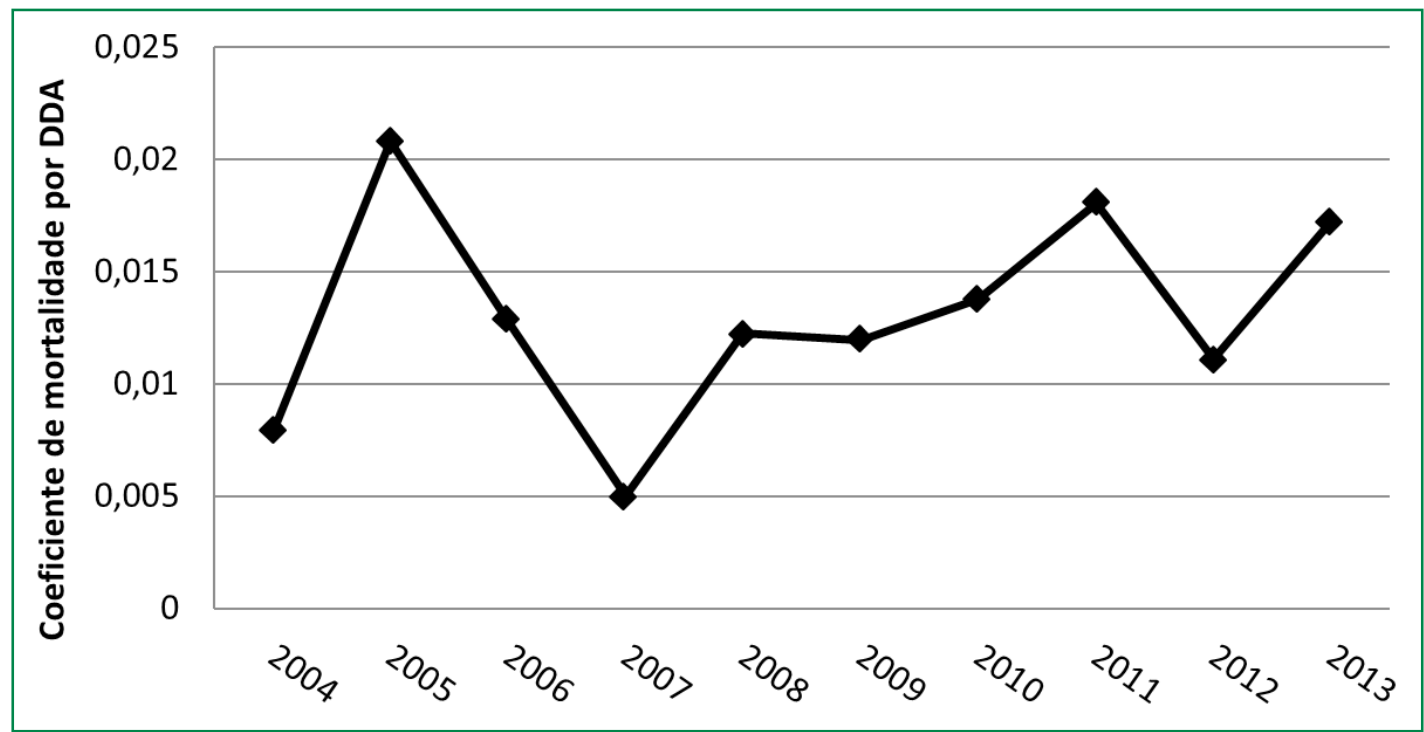

Figura 5. Coeficiente de mortalidade por doença diarreica aguda no Município de Caxias do Sul, Rio Grande do Sul, Brasil, 2004-2013.

\section{DISCUSSÃO}

A avaliação de um agravo por longos períodos tem grande aplicabilidade na gestão pública de um município. O acompanhamento dos casos de DDA registrados por 10 anos, em Caxias do Sul, mostrou um discreto aumento de casos a partir do ano de 2005 e não foi verificada uma sazonalidade para essa doença. Apesar de as gastroenterites apresentarem-se de forma endêmica, cinco surtos epidêmicos acometeram a população nesse período, os quais ocorreram principalmente nos meses de verão dos anos de 2010 e 2011 e nos meses de inverno de 2004 e 2012. Na literatura mundial, a correlação sazonal para as DDA não é amplamente estudada. Este fato se justifica por se tratar de uma doença multifatorial. Contudo, a alta incidência de gastroenterites por rotavírus é bem relacionada aos períodos de inverno [19]. Estudos de sazonalidade confirmam esses resultados nas Regiões Sudeste e Central do Brasil, onde o inverno se caracteriza por climas mais secos e os verões são chuvosos [20-22]. Apesar de o rotavírus ser o principal agente causador de diarreias, o presente estudo não pesquisou os agentes etiológicos das gastroenterites, e é possível que os surtos nos meses de verão tenham sido provocados por outros agentes que não o rotavírus. Os achados sobre a sazonalidade indicam a necessidade de se estudar a relação do período do ano com os diferentes agentes etiológicos separadamente.

O clima de Caxias do Sul conta com uma temperatura média de $16,5^{\circ} \mathrm{C}$ e a precipitação pluvio- métrica anual é de $1.915 \mathrm{~mm}$ [18]. As chuvas são regularmente distribuídas durante o ano. Em geral, a maior precipitação pluviométrica encontra-se no mês de março (206 mm) e o mês menos chuvoso é maio (109 mm). É, portanto, uma região úmida. Em nosso estudo, verificou-se uma correlação inversa, porém não significativa, entre a alta incidência de DDA e períodos de precipitação pluviométrica. Tal correlação poderia ser explicada pela constante pluviosidade da região da serra gaúcha, onde a chuvas distribuem-se de forma regular nas diferentes estações do ano. Entretanto, para obter dados significativos e concluir sobre este aspecto, talvez fosse necessária a análise de incidência de DDA com os dados de sucessivos de dias chuvosos ou secos. Adicionalmente, estudo realizado em Goiânia [20] correlacionou um dos agentes etiológicos da DDA aos períodos de seca regional. Nesse trabalho, os autores demonstram que as infecções por rotavírus são mais frequentes nos períodos de menor pluviosidade. Possivelmente, ambientes mais secos favorecem a disseminação aérea das partículas virais. E, apesar de existirem outros agentes etiológicos envolvidos, os vírus têm uma contribuição muito expressiva na DDA [20]. Outro trabalho identificou um aumento nos casos de diarreia nos meses de janeiro a março, que correspondem ao período de chuvas na Região Norte do País, o que pode estar relacionado à contaminação do lençol freático ou a ingestão de águas de fontes diferentes das habituais [9]. Os períodos de chuva podem estar implicados com os agentes etiológicos de veiculação hídrica, como protozoários e vírus [21,22]. 
Muito já se sabe sobre a participação de norovírus, adenovírus e sapovírus em surtos gastroentéricos oriundos de água potável e água de recreação [11,22]. Porém, deve-se considerar a heterogeneidade brasileira: por ser um país de dimensões continentais e de regiões com extremas diferenças ambientais, climáticas e culturais, é de se esperar que se encontrem dados totalmente distintos. Na Região Sul, apesar de ser a única a apresentar um frio intenso no inverno, tem ocorrido verões extremamente quentes nos últimos anos.

O predomínio dos registros de gastroenterites ocorreu em regiões da periferia da cidade de Caxias do Sul. Os bairros destacados foram Desvio Rizzo, Reolon e Santa Fé, sendo estes dois últimos os mais populosos do município e conhecidos por terem baixos indicadores sociais e apresentarem altos índices de pobreza. Sabe-se que as baixas condições de saneamento básico propiciam a disseminação de doenças [23]. Adicionalmente, estudos demonstram que os cidadãos de bairros de menor poder aquisitivo procuram frequentemente as unidades de pronto atendimento, o que pode contribuir para um maior número de registros [2].

Ao longo do período estudado, observou-se um decréscimo nas internações hospitalares de crianças de zero a quatro anos decorrentes de DDA em Caxias do Sul. Resultados semelhantes foram encontrados nas Cidades de Manaus, Vitória e Brasília, onde as taxas de internações infantis foram descendentes entre 1995 e 2005 [24]. A redução das admissões hospitalares em Caxias do Sul possivelmente é explicada pelo impacto do programa de vacinação contra rotavírus, implantado no Brasil a partir de março de 2006, que protegeu imunologicamente parte da população envolvida neste estudo de um dos agentes etiológicos mais expressivos da DDA $[4,25,26]$.

Duas novas vacinas contra o rotavírus têm demonstrado eficácia em grandes ensaios clínicos. Entre 2006 e 2010, 27 países introduziram a vacinação, e posteriormente, os índices de doenças graves causadas por rotavírus diminuíram em crianças vacinadas e não vacinadas. Pesquisas sustentam que a vacinação contra o rotavírus tem levado a grandes declínios da mortalidade infantil por diarreia no Brasil e México [4]. O programa de vacinação contra rotavírus, iniciado em
2006 em Caxias do Sul, logo alcançou a meta esperada, atingindo mais de $90 \%$ da população de crianças imunizadas ainda em 2007.

O impacto da vacinação contra rotavírus não foi percebido nos coeficientes de mortalidade (em todas as faixas etárias) em Caxias do Sul, o que corrobora com o que é descrito em vários trabalhos publicados no Brasil e em outros países [25]. Notou-se uma flutuação no número de casos que poderia ser explicada pelo aumento populacional de Caxias do Sul, que passou de 360.419 habitantes no ano de 2000 para 470.223 em 2014 [17]. Sabe-se que o aumento da população do município é devido basicamente a migrantes, que vêm de outras regiões do Estado e do País, em busca de melhores condições de trabalho. Em paralelo, é notório que o aumento de registros de óbitos pode ter advindo do maior acesso à informatização, facilitando os registros pelos profissionais que atuaram no atendimento nesse período de 10 anos [9].

$O$ registro de mortalidade infantil por diarreia aguda vem declinando em quase todo o território brasileiro. Entre os fatores que têm contribuído para esse declínio estão o soro de reidratação oral, o aleitamento materno, as melhores condições de saúde da população, a diminuição da desnutrição infantil e as melhorias na infraestrutura de saneamento básico. Nos países mais pobres, a diarreia é a causa mais comum de morte em crianças menores de cinco anos de idade, sendo que das 13.700 pessoas que morrem diariamente por doenças transmitidas pela água, mais da metade são crianças $[3,5,24,27,28]$.

O estudo desta série histórica de 10 anos pode servir de indicador epidemiológico. A avaliação da incidência de DDA, por esse longo período, mostra que houve flutuações ao longo do tempo no número de registros da doença, assim como de óbitos em Caxias do Sul. Este estudo revelou também uma redução no número de internações devidas à DDA no período que sucedeu à introdução do programa de vacinação contra rotavírus na rede pública de saúde. Estes resultados podem contribuir para ampliar os conhecimentos da incidência de DDA, permitindo o monitoramento do agravo, indicando possíveis formas de prevenção e contribuindo para o planejamento de futuras ações em saúde do município.

\section{AGRADECIMENTOS}

A Jacqueline Silva de Oliveira, Rejane Fátima Rech e Ivanise Susin dos Santos, do Setor de Vigilância Epidemiológica da Secretaria Municipal de Saúde de Caxias do Sul, pelo levantamento e disponibilização dos dados referentes à doença diarreica aguda; ao Professor Dr. Petrônio Fagundes de Oliveira Filho, pelo auxílio às análises dos dados; ao biólogo Cassiano Alves Marchett, pela confecção do mapa; e à Universidade de Caxias do Sul. 


\section{REFERÊNCIAS}

1. Rackoff LA, Bok K, Green KY, Kapikian AZ. Epidemiology and evolution of rotaviruses and noroviruses from archival WHO global study in children (1976-79) with implications for vaccine design. Plos One. 2013;8(3):1-12. http://dx.doi.org/10.1371/journal.pone.0059394

2. Facanha MC, Pinheiro AC. Comportamento das doenças diarreicas agudas em serviços de saúde de Fortaleza, Ceará, Brasil, entre 1996 e 2001. Cad Saude Publica. 2005;21(1):49-54. http://dx.doi.org/10.1590/S0102-311X2005000100006

3. Victora CG. Diarrhea mortality: what can the world learn from Brazil? J Pediatr. 2009;85(1):3-5. http://dx.doi.org/10.2223/JPED.1860

4. Patel MM, Glass RI, Desai R, Tate JE. Fulfilling the promise of rotavirus vaccines: haw far have we come since licensure? Lancet Infect Dis. 2012;12(7):561-70. http://dx.doi.org/10.1016/S1473-3099(12)70029-4

5. Parashar UD, Hummelman EG, Bresee JS, Miller MA, Glass RI. Global illness and deaths caused by rotavirus disease in children. Emerg Infect Dis. 2003;9(5):565-72. http://dx.doi.org/10.3201/eid0905.020562

6. Junqueira RM, Duarte EC. Hospitalizations due to ambulatory care-sensitive conditions in the Federal District, Brazil, 2008. Rev Saude Publica. 2012;46(5):761-8. http://dx.doi.org/10.1590/S0034-89102012000500001

7. Luchs A, Morillo SG, Ribeiro CD, Cilli A, Calux SJ, Carmona RCC, Timenetsky, MCST. Rotavirus G2P[4] and G2P[4]+[6] infections during norovirus gastroenteritis outbreak: summer season 2010, Brazil. Rev Soc Bras Med Trop. 2013;46(2):227-30. http://dx.doi. org/10.1590/0037-8682-1162-2013

8. Platts-Mills JA, Liu J, Houpt ER. New concepts in diagnostics for infectious diarrhea. Mucosal Immunol. 2013;6(5):876-85. http://dx.doi. org/10.1038/mi.2013.50

9. Dias DM, Silva AP, Helfer AM, Maciel AMTR, Loureiro ECB, Souza CO. Morbimortalidade por gastroenterites no Estado do Pará. Revista Pan-Amazônica de Saúde. 2010;1(1):53-60. http://dx.doi.org/10.5123/S2176-62232010000100008

10. Almeida MT, Silva RM, Donaire LM, Moreira LE, Martinez MB. Enteropatógenos associados com diarreia aguda em crianças. J Pediatr. 1998;74:291-8. http://dx.doi.org/10.2223/JPED.444

11. Glass RI. Beyond discovering the viral agents of acute gastroenteritis. Emerg Infect Dis. 2013;19(8):1190-1. http://dx.doi.org/10.3201/ eid1908.130773

12. Medeiros MI, Neme SN, da Silva P, Capuano DM, Errera MC, Fernandes SA, Valle GR, Avila FA. Etiology of acute diarrhea among children in Ribeirao Preto-SP, Brazil. Rev Inst Med TropSao Paulo. 2001;43(1):21-4. http://dx.doi.org/10.1590/s0036-46652001000100004

13. Moresco V, Viancelli A, Nascimento MA, Souza DS, Ramos AP, Garcia LA, Simões CM, Barardi CR. Microbiological and physicochemical analysis of the coastal waters of southern Brazil. Mar Pollut Bull. 2012;64(1):40-8. http://dx.doi.org/10.1016/j.marpolbul.2011.10.026

14. Nascimento WR, Cavalcanti IM, Irmao JI, Rocha FJS. Presença de Cryptosporidium spp em crianças com diarreia aguda em uma creche pública de Recife, Estado de Pernambuco. Rev Soc Bras Med Trop. 2009;42(2):175-8. http://dx.doi.org/10.1590/S0037-86822009000200016

15. Brasil. Ministério da Saúde (MS). Departamento de In $\neg$ formática do SUS (DATASUS). Sistema de Informação de Mortalidade (SIM). Indicadores de mortalidade, 2011 [Internet]. [cited 2015 April]. Available from http://tabnet.datasus.gov.br/cgi/tabcgi.exe?idb2012/c06.def

16. Ambrosini VA, Carraro E. Impacto da vacinação contra rotavírus no Brasil. Medicina (Ribeirão Preto). 2012;45(4):411-18.

17. IBGE. Instituto Brasileiro de Geografia e Estatística. IBGE cidades 2014 [Internet]. [Cited 2015 April 10]. Available from: http://www. cidades.ibge.gov.br/xtras/home.php

18. WregeMS, Steinmetz S, Matzenauer R, RadinB, AlmeidaIR, Reisser Junior C, Maluf JRT, Bueno AC, Pasinato A, Cunha GR, Prestes SD, Dalmago GA, Pires JLF, Santi A, Berlato MA, Didoné IA. Atlas climático do Rio Grande do Sul [Internet]. Porto Alegre; CEMETRS/ FEPAGRO 2013. [cited 2015 April]. Available from: www.cemet.rs.gov.br/area/7/Atlas_Climático

19. Hashizume M, Armstrong B, Wagatsuma Y, Faruque AS, Hayashi T, Sack DA. Rotavirus infections and climate variability in Dhaka, Bangladesh: a time-series analysis. Epidemiol Infect. 2008;136(9):1281-9. http://dx.doi.org/10.1017/s0950268807009776

20. Cardoso DDP, Martins IRMB, Kitajima EW, Camarota ISCT, Azevedo IMSP. Rotavírus e adenovírus em crianças de 0-5 anos hospitalizadas com ou sem gastroenterite em Goiânia-GO, Brasil. Rev Inst Med Trop São Paulo. 1992;34(5):433-9. http://dx.doi.org/10.1590/S003646651992000500010

21. Teixeira JC, Heller L. Impact of water supply, domiciliary water reservoirs and sewage on faeco-orally transmitted parasitic diseases in children residing in poor areas in Juiz de Fora, Brazil. Epidemiol Infect. 2006;134(4):694-8. http://dx.doi.org/10.1017/S0950268805005443

22. Bruggink LD, Marshall JA. The incidence of norovirus-associated gastroenteritis outbreaks in Victoria, Australia (2002-2007) and their relationship with rainfall. Int J Environ Res Public Health. 2010;7(7):2822-7. http://dx.doi.org/10.3390/ijerph7072822

23. Spilki FR, da Luz RB, Fabres RB, Soliman MC, Kluge M, Fleck JD, Rodrigues MT, Comerlato J, Cenci A, Cerva C, Dasso MG, Roehe PM. Detection of human adenovirus, rotavirus and enterovirus in water samples collected on dairy farms from Tenente Portela, Northwest of Rio Grande do Sul, Brazil. Braz J Microbiol. 2013;44(3):953-7. http://dx.doi.org/10.1590/S1517-83822013000300046

24. Oliveira TC, Latorre M do R. Tendências da internação e da mortalidade infantil por diarreia: Brasil, 1995 a 2005. Rev Saúde Publica 2010;44(1):102-11. http://dx.doi.org/10.1590/S0034-89102010000100011

25. Linhares AC, Justino MC. Rotavirus vaccination in Brazil: effectiveness and health impact seven years post-introduction. Expert Rev Vaccines. 2014;13(1):43-57. http://dx.doi.org/10.1586/14760584.2014.861746

26. Luchs A, Cilli A, Morillo SG, de Cassia Compagnoli Carmona R, do Carmo Sampaio Tavares Timenetsky M. Rotavirus in adults, Brazil, 2004-2011: G2P[4] dominance and potential impact on vaccination. Braz J Infect Dis. 2014;18(1):53-9. http://dx.doi.org/10.1016/j. bjid.2013.05.010

27. Portela RA, Leite VD, Pereira CF, Rocha EMFM. Comportamento das doenças diarreicas nas mudanças sazonais no município de Campina Grande-PB. Hygeia. 2013;9(17):116-28.

28. Braga B, Conejo JGL, Hespanhol I, Mierzwa JC. Introdução à Engenharia Ambiental: Conhecimento especializado - Saneamento e Tecnologia Ambiental. $2^{\mathrm{a}}$ ed. São Paulo: Prentice Hall; 2002. 